\title{
DISTÚRBIOS PSÍQUICOS MENORES EM TRABALHADORES DE ENFERMAGEM DE UM BLOCO CIRÚRGICO
}

\author{
MINOR PSYCHOLOGICAL DISORDERS \\ IN NURSING WORKERS IN A SURGICAL WARD
}

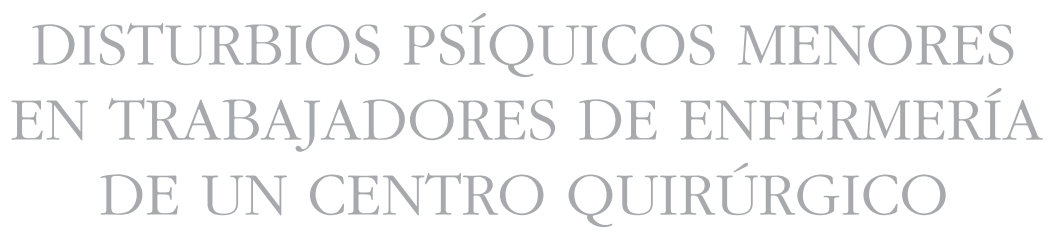

Lauren Leal Ferreira ${ }^{1}$

Evelin Daiane Gabriel Pinhatti ${ }^{2}$

Cinthya Kallyane Gonçalves Queiroz ${ }^{3}$

Renata Perfeito Ribeiro ${ }^{4}$

Como citar este artigo: Ferreira LL, Pinhatti EDG, Queiroz CKG, Ribeiro RP. Distúrbios Psíquicos Menores em trabalhadores de enfermagem de um Bloco Cirúrgico. Rev baiana enferm. 2019;33:e28279.

Objetivo: analisar a proporção e os fatores associados aos Distúrbios Psíquicos Menores em trabalhadores de enfermagem de um Bloco Cirúrgico. Método: estudo descritivo, exploratório, de abordagem quantitativa, realizado com 54 trabalhadores de enfermagem. Para a coleta de dados, utilizou-se questionário estruturado contendo características sociodemográficas e ocupacionais, a versão brasileira do Self Reporting Questionnaire-20 e o Demand Control Support Questionnaire. Resultados: a proporção de Distúrbios Psíquicos Menores foi de 24,1\%. Em relação aos aspectos psicossociais, foram predominantemente classificados como desenvolvendo um trabalho com baixa demanda psicológica $(63,0 \%)$, baixo controle $(70,4 \%)$ e baixo apoio social $(72,2 \%)$. Das variáveis investigadas, apresentaram associação estatisticamente significativa com o desfecho o sexo feminino e o controle no trabalho. Conclusão: os resultados evidenciaram a proporção de 24,1\% para os Distúrbios Psíquicos Menores nos trabalhadores de enfermagem do local estudado; quanto aos fatores associados, o sexo feminino e o controle no trabalho apresentaram associação significativa com o desfecho.

Descritores: Centro Cirúrgico. Enfermagem. Saúde do Trabalhador. Trabalhadores. Transtornos Mentais.

Objective: analyzing the proportion of Minor Psychological Disorders in nursing workers in a Surgical Ward and the factors associated with these disorders. Method: descriptive, exploratory, and quantitative study, carried out with 54 nursing workers. For data collection, this study used a structured questionnaire with sociodemographic and occupational characteristics, the Brazilian version of the Self-Reporting Questionnaire-20 and the Demand Control Support Questionnaire. Results: the proportion of Minor Psychological Disorders was 24.1\%. The psychosocial aspects were mostly classified as developing a work with a low psychological burden (63.0\%), low control (70.4\%), and low social support (72.2\%). The variables female sex and control at work presented statistically significant correlations to the outcome. Conclusion: the results have shown the proportion of 24.1\% of Minor Psychological Disorders among

\footnotetext{
Enfermeira. Pesquisadora independente. Londrina. Paraná. Brasil. lauren.leal96@gmail.com.

Enfermeira. Mestra em Enfermagem. Pesquisadora independente. Londrina. Paraná. Brasil.

Enfermeira. Enfermeira do trabalho na empresa Belagrícola Comércio e Representações de Produtos Agrícolas S.A. Londrina, Paraná, Brasil.

Enfermeira. Pós-doutora em Enfermagem. Docente adjunto do Curso de Enfermagem da Universidade Estadual de Londrina. Londrina, Paraná, Brasil.
} 
nursing workers in the setting of the study. Regarding associated factors, the female sex and control at work presented a significant association with the outcome.

Descriptors: Surgicenter. Nursing. Occupational Health. Workers. Mental Disorders.

Objetivo: analizar la proporción de Disturbios Psíquicos Menores en trabajadores de enfermería de un Centro Quirúrgico y factores asociados a eses Disturbios. Método: estudio descriptivo, exploratorio, de abordaje cuantitativo, con la participación de 54 trabajadores de enfermería. Para la colección de los datos, se utilizó un cuestionario estructurado conteniendo características sociodemográficas y ocupacionales, la versión brasileña del Self-Reporting Questionnaire-20 y el Demand Control Support Questionnaire. Resultados: la proporción de Disturbios Psíquicos Menores fue de $24.1 \%$. Con respecto a los aspectos psicosociales, se los clasificó mayoritariamente como el desarrollo de un trabajo con bajas exigencias psicológicas (63.0\%), poco control (70.4\%), y poco apoyo social. Entre las variables investigadas, el sexo femenino y el control en el trabajo presentaron asociación estadísticamente significativa con el resultado. Conclusión: los Disturbios Psíquicos Menores presentaron una proporción de 24.1\% entre los trabajadores de enfermería del sitio estudiado; con respecto a los factores asociados, el sexo femenino y el control en trabajo presentaron asociación significativa con los resultados.

Descriptores: Centro Quirúrgico. Enfermería. Salud del Trabajador. Trabajadores. Trastornos Mentales.

\section{Introdução}

Nas primeiras décadas deste século XXI, o mercado de trabalho sofreu grande expansão no tocante aos fatores relacionados à economia, ao aumento das tecnologias e do acesso à informação e às inovações. A preocupação resultante dessas mudanças com relação à saúde e à segurança dos trabalhadores em seus ambientes laborais têm conduzido pesquisadores a desenvolverem estudos mais aprofundados sobre essa problemática $^{(1)}$.

$\mathrm{Na}$ área da saúde, estudos demonstram que os profissionais estão expostos a diversos riscos ocupacionais que podem afetar sua saúde física e mental. Esses riscos estão relacionados às exposições a um cotidiano cada vez mais estressante, com rotinas exaustivas, condições inapropriadas de trabalho, convívio com a dor, o sofrimento e a falta de recursos físicos e materiais que favoreçam o trabalho ${ }^{(2-3)}$.

Nesse cenário, as categorias de enfermagem são as mais referidas, pelo contato contínuo com sofrimento, morte, jornadas de trabalho múltiplas, sobrecarga de trabalho e remuneração desproporcional ao trabalho que executam. Como consequência, esses trabalhadores estão suscetíveis ao adoecimento físico e psíquico ${ }^{(4)}$.

Pesquisas apontam que, em setores críticos e fechados, como Bloco Cirúrgico (BC), composto por Centro Cirúrgico (CC), Sala de Recuperação Anestésica (SRA) e Central de Materiais e Esterilização (CME), existe maior propensão para o adoecimento dos que ali trabalham. Isso se justifica devido às características desses locais, como iluminação natural escassa, acesso restrito de pessoas, excesso de barulho dos equipamentos, entre outras, que aumentam ao longo do tempo o risco para o desenvolvimento de sintomas prejudiciais à saúde do trabalhador ${ }^{(5-6)}$.

Os trabalhadores que atuam no CC e na SRA estão imersos em um ambiente onde há alta cobrança para o conhecimento tecnológico e agilidade no processo de trabalho. Estudos apontam relações fortes de hierarquia que podem favorecer as relações de poder e acarretar conflitos internos, além dos riscos inerentes à exposição física e consequentes agravos osteomusculares ${ }^{(7-8)}$. Ainda atrelado a esses problemas, estão as poucas horas de descanso em decorrência da alta demanda dos trabalhadores que atuam no ambiente perioperatório ${ }^{(9)}$.

A CME, apesar de ser fundamental dentro dos serviços de saúde para reduzir as taxas de infecções, é considerada uma unidade prestadora de assistência indireta ao paciente. Esse entendimento resulta em menor valorização do trabalho e leva à ideia de invisibilidade do que 
é produzido. Diante disso, há uma cultura de que grande parte dos trabalhadores que estão inaptos para os cuidados com pacientes, possuem relações complexas com a equipe, dificuldades de aprendizado e restrições físicas no trabalho sejam remanejados para a $\mathrm{CME}^{(10)}$.

No que se refere ao adoecimento mental, os Distúrbios Psíquicos Menores (DPM) caracterizam-se por sintomas depressivos e ansiosos, como tristeza, fadiga, diminuição da concentração, irritabilidade, insônia e queixas somáticas ${ }^{(11)}$. Os DPM vêm sendo mencionados frequentemente na literatura como causadores do adoecimento mental em trabalhadores, principalmente em pesquisas realizadas no ambiente hospitalar. Estudos realizados no Rio Grande do Sul e na Bahia apontam prevalência de 33,7\% e $35,0 \%$, respectivamente, desses distúrbios nesse ambiente $^{(12-13)}$.

Diante do pressuposto de que os trabalhadores que atuam no BC estão expostos às condições que favorecem o desenvolvimento de sintomas prejudiciais à saúde mental, considera-se relevante investigar como se apresenta a saúde mental desses trabalhadores, pois o conhecimento da situação atual de saúde pode auxiliar na elaboração de medidas para intervenção na prática.

Assim, este estudo tem como objetivo analisar a proporção e os fatores associados aos Distúrbios Psíquicos Menores em trabalhadores de enfermagem de um Bloco Cirúrgico.

\section{Método}

Estudo descritivo, exploratório, de abordagem quantitativa, realizado em um Hospital Universitário de grande porte do estado do $\mathrm{Pa}$ raná, Brasil.

A população do estudo constituiu-se de 71 trabalhadores de enfermagem que atuavam no BC. O critério de inclusão foi ser trabalhador da área de enfermagem do BC em atividade. O critério de exclusão foi ter retornado ao trabalho há menos de 30 dias (critério de um dos instrumentos utilizados nesta pesquisa). Assim, 62 trabalhadores preencheram os critérios de elegibilidade, mas, desses, 8 recusaram-se a participar do estudo. Para o cálculo do tamanho amostral, aplicou-se a seguinte fórmula utilizada para população finita:

$n=N \cdot p \cdot q \cdot(\mathrm{Z \alpha} / 2)^{2} /(N-1) \cdot(E)^{2}+$ p.q. $(\mathrm{Z \alpha} / 2)^{2}$

A amostra foi estratificada por categoria profissional e calculada utilizando-se intervalo de confiança de $95 \%$, erro máximo de $5 \%$ e proporção de $50 \%$ que resulta no maior número amostral. Assim, obteve-se o quantitativo mínimo de 7 enfermeiros, 18 auxiliares de enfermagem e 29 técnicos de enfermagem.

Para levantamento dos dados, foi utilizado um questionário estruturado contendo questões referentes às características sociodemográficas e ocupacionais dos trabalhadores. Para mensuração dos DPM, foi aplicada a versão brasileira do questionário Self Reporting Questionnaire (SRQ-20), com 20 questões dicotômicas para rastreamento de transtornos mentais não-psicóticos, que avaliam sintomas depressivos, somáticos e de ansiedade ocorridos nos últimos 30 dias. $\mathrm{O}$ ponto de corte utilizado foi de sete ou mais respostas positivas ${ }^{(14)}$.

A coleta de dados foi realizada no período de novembro de 2016 a janeiro de 2017. Os questionários foram entregues e recolhidos por uma das autoras no local de trabalho dos participantes, após esclarecimento dos objetivos da pesquisa e assinatura do Termo de Consentimento Livre e Esclarecido (TCLE).

Os aspectos psicossociais foram avaliados com o auxílio do Demand Control Support Questionnaire (DCSQ), adaptado por Töres Theorell em $1988^{(15)}$. A versão brasileira é composta por 17 questões, com três dimensões que avaliam demanda psicológica, controle no trabalho e apoio social. Dentre as cinco perguntas que avaliam a demanda psicológica, quatro referem-se ao tempo e à velocidade para realização do trabalho e uma avalia os conflitos existentes entre as diferentes demandas. Das seis questões referentes ao controle no trabalho, quatro referem-se ao uso e desenvolvimento de habilidades e duas à autonomia para tomada de decisão no processo 
de trabalho. Seis questões avaliam o apoio social entre chefia e colegas ${ }^{(16)}$. As dimensões foram categorizadas em (alta/baixa) tendo como ponto de corte a mediana, após verificação da distribuição assimétrica dos dados.

Para a análise dos dados, utilizou-se o programa Statistical Package for the Social Sciences (SPSS), versão 20.0. Para caracterização da amostra, foram realizadas análises descritivas por meio de frequência absoluta e relativa para as variáveis categóricas; para as varáveis contínuas, foram realizadas medidas de tendência central e dispersão. Para associações entre os DPM (variável dependente) e as variáveis sociodemográficas, ocupacionais e o modelo DCSQ, foi utilizado o teste Qui-quadrado, considerando associação significativa as variáveis que apresentaram $\mathrm{p}<0,05$. Vale ressaltar que, nas análises, a proporção foi utilizada como medida de ocorrência, e a diferença entre as proporções, como medida de associação.

Esta pesquisa atendeu às recomendações da Resolução no 466, de 12 de dezembro de 2012, com aprovação Comitê de Ética em Pesquisa, sob Certificado de Apresentação para Apreciação Ética (CAAE) n 58056916.0.0000.5231.

\section{Resultados}

A amostra estudada foi composta por 54 trabalhadores de enfermagem. As perdas resultantes de recusas totalizaram 12,9\%. A proporção de suspeição de Distúrbios Psíquicos Menores foi de 24,1\%. Na Tabela 1 são apresentadas as características sociodemográficas e ocupacionais e a associação com os DPM.

Tabela 1 - Características sociodemográficas e ocupacionais e associação com Distúrbios Psíquicos Menores em trabalhadores de enfermagem de um bloco cirúrgico. Paraná, Brasil 2016-2017 $(n=54)$

(continua)

\begin{tabular}{|c|c|c|c|c|}
\hline \multirow{2}{*}{$\begin{array}{l}\text { Características Sociodemográficas e } \\
\text { Ocupacionais }\end{array}$} & \multirow[t]{2}{*}{ n $(\%)$} & \multicolumn{2}{|c|}{$\begin{array}{c}\text { Distúrbios Psíquicos } \\
\text { Menores }\end{array}$} & \multirow[t]{2}{*}{ p-valor ${ }^{(1)}$} \\
\hline & & Sim & Não & \\
\hline \multicolumn{5}{|l|}{ Sexo } \\
\hline Feminino & $44(81,5)$ & 13 & 31 & \multirow[t]{2}{*}{0,049} \\
\hline Masculino & $10(18,5)$ & - & 10 & \\
\hline \multicolumn{5}{|l|}{ Idade } \\
\hline $30-39$ anos & $11(20,3)$ & 3 & 8 & \multirow{4}{*}{0,854} \\
\hline $40-49$ anos & $23(42,6)$ & 6 & 17 & \\
\hline $50-59$ anos & $18(33,3)$ & 4 & 14 & \\
\hline$>60$ & $2(3,8)$ & - & 2 & \\
\hline \multicolumn{5}{|l|}{ Situação Conjugal } \\
\hline Solteiro & $9(16,7)$ & 1 & 8 & \multirow[t]{3}{*}{0,603} \\
\hline Casado/União estável & $32(59,2)$ & 8 & 24 & \\
\hline Divorciado/Desquitado/Viúvo & $13(24,1)$ & 4 & 9 & \\
\hline \multicolumn{5}{|l|}{ Filhos } \\
\hline Sim & $46(85,2)$ & 11 & 35 & \multirow[t]{2}{*}{0,947} \\
\hline Não & $8(14,8)$ & 2 & 6 & \\
\hline \multicolumn{5}{|l|}{ Nivel de instrução } \\
\hline Ensino técnico & $3(5,6)$ & - & 3 & \multirow{4}{*}{0,633} \\
\hline Ensino superior & $26(48,2)$ & 7 & 19 & \\
\hline Especialização & $21(38,8)$ & 6 & 15 & \\
\hline Mestrado/Doutorado & $4(7,4)$ & - & 4 & \\
\hline \multicolumn{5}{|l|}{ Função } \\
\hline Auxiliar de enfermagem & $18(33,3)$ & 6 & 12 & \multirow[t]{3}{*}{0,216} \\
\hline Técnico de enfermagem & $29(53,7)$ & 7 & 22 & \\
\hline Enfermeiro & $7(13,0)$ & - & 7 & \\
\hline
\end{tabular}


Tabela 1 - Características sociodemográficas e ocupacionais e associação com Distúrbios Psíquicos Menores em trabalhadores de enfermagem de um bloco cirúrgico. Paraná, Brasil 2016-2017 ( $n=54)$

(conclusão)

\begin{tabular}{|c|c|c|c|c|}
\hline \multirow{2}{*}{$\begin{array}{l}\text { Características Sociodemográficas e } \\
\text { Ocupacionais }\end{array}$} & \multirow{2}{*}{ n (\%) } & \multicolumn{2}{|c|}{$\begin{array}{l}\text { Distúrbios Psíquicos } \\
\text { Menores }\end{array}$} & \multirow{2}{*}{ p-valor ${ }^{(1}$} \\
\hline & & Sim & Não & \\
\hline \multicolumn{5}{|l|}{ Tempo na instituição } \\
\hline Até 5 anos & $5(9,3)$ & - & 5 & 0,353 \\
\hline $6-10$ anos & $9(16,7)$ & 3 & 6 & \\
\hline $11-20$ anos & $18(33,3)$ & 3 & 15 & \\
\hline$>20$ anos & $22(40,7)$ & 7 & 15 & \\
\hline \multicolumn{5}{|l|}{ Turno } \\
\hline Diurno & $38(70,4)$ & 9 & 29 & 0,918 \\
\hline Noturno & $16(29,6)$ & 4 & 12 & \\
\hline \multicolumn{5}{|l|}{$\operatorname{Renda}^{(2)}(\mathbf{n}=\mathbf{3 2})^{(3)}$} \\
\hline Até 4 salários & $8(25,0)$ & 3 & 5 & 0,304 \\
\hline 4 - 7 salários & $13(40,6)$ & 4 & 9 & \\
\hline >7 salários & $11(34,4)$ & 1 & 10 & \\
\hline
\end{tabular}

Fonte: Elaboração própria.

Nota: Sinal convencional utilizado:

- Dado numérico igual a zero não resultante de arredondamento.

(1) p-valor: teste qui-quadrado;

(2) Salário mínimo no ano de 2017: R \$937,00;

(3) Participantes que responderam a questão sobre renda.

Observou-se, entre os trabalhadores com suspeição de DPM, que 53,8\% foram classificados com baixa demanda psicológica, 53,8\% com alto controle no trabalho e $84,6 \%$ com baixo apoio social. Na análise de associação, apenas o controle no trabalho manteve-se associado aos DPM, conforme apresentado na Tabela 2.

Tabela 2 - Associação entre aspectos psicossociais do trabalho e Distúrbios Psíquicos Menores entre trabalhadores de enfermagem de um bloco cirúrgico. Paraná, Brasil - 2016-2017 (n=54)

\begin{tabular}{|c|c|c|c|c|}
\hline \multirow{2}{*}{$\begin{array}{l}\text { Modelo demanda-controle e } \\
\text { apoio social }\end{array}$} & \multirow{2}{*}{ n (\%) } & \multicolumn{2}{|c|}{ Distúrbios Psíquicos Menores } & \multirow{2}{*}{ p-valor ${ }^{(1)}$} \\
\hline & & Sim & Não & \\
\hline \multicolumn{5}{|l|}{ Demanda } \\
\hline Baixa & $34(63,0)$ & 7 & 27 & 0,435 \\
\hline Alta & $20(37,0)$ & 6 & 14 & \\
\hline \multicolumn{5}{|l|}{ Controle } \\
\hline Baixo & $38(70,4)$ & 6 & 32 & 0,028 \\
\hline Alto & $16(29,6)$ & 7 & 9 & \\
\hline \multicolumn{5}{|l|}{ Apoio social } \\
\hline Baixo & $39(72,2)$ & 11 & 28 & 0,219 \\
\hline Alto & $15(27,8)$ & 2 & 13 & \\
\hline
\end{tabular}

Fonte: Elaboração própria.

(1) p-valor: teste qui-quadrado. 
Na Tabela 3, apresentam-se as frequências de respostas afirmativas e os grupos de sintomas do SRQ-20. A média das respostas afirmativas do SRQ-20 foi de $4,8( \pm 3,8)$, com mínimo de zero e máximo de quinze. O grupo de sintomas que obteve mais respostas positivas foram os sintomas somáticos $(75,9 \%)$, em segundo o humor depressivo-ansioso (72,2\%), seguidos de decréscimo de energia vital $(64,8 \%)$ e pensamento depressivo $(18,5 \%)$.

Tabela 3 - Distribuição das respostas de acordo com os grupos do SRQ-20 entre os trabalhadores de enfermagem de um bloco cirúrgico. Paraná, Brasil - 2016-2017 (n=54)

\begin{tabular}{|c|c|c|}
\hline Grupos de Sintomas & $\mathbf{n}$ & $(\%)$ \\
\hline \multicolumn{3}{|l|}{ Humor Depressivo-ansioso } \\
\hline Sente-se nervoso(a), tenso(a) ou preocupado(a) & 28 & $(51,8)$ \\
\hline Assusta-se com facilidade & 18 & $(33,3)$ \\
\hline Tem se sentido triste ultimamente & 19 & $(35,1)$ \\
\hline Tem chorado mais do que costume & 7 & $(12,9)$ \\
\hline \multicolumn{3}{|l|}{ Sintomas Somáticos } \\
\hline Tem dores de cabeça frequentes & 27 & $(50,0)$ \\
\hline Dorme mal & 23 & $(42,5)$ \\
\hline Tem sensações desagradáveis no estômago & 21 & $(38,8)$ \\
\hline Tem má digestão & 19 & $(35,1)$ \\
\hline Tem falta de apetite & 4 & $(7,4)$ \\
\hline Tem tremores nas mãos & 4 & $(7,4)$ \\
\hline \multicolumn{3}{|l|}{ Decréscimo de Energia Vital } \\
\hline Cansa com facilidade & 19 & $(35,1)$ \\
\hline Tem dificuldades para tomar decisões & 7 & $(12,9)$ \\
\hline Encontra dificuldade para realizar com satisfação as atividades diárias & 15 & $(27,7)$ \\
\hline Tem dificuldades no serviço (seu trabalho é penoso, lhe causa sofrimento) & 4 & $(7,4)$ \\
\hline Sente-se cansado o tempo todo & 18 & $(33,3)$ \\
\hline Tem dificuldade de pensar com clareza & 11 & $(20,3)$ \\
\hline \multicolumn{3}{|l|}{ Pensamento Depressivo } \\
\hline É incapaz de desempenhar um papel útil em sua vida & 2 & $(3,7)$ \\
\hline Tem perdido o interesse pelas coisas & 8 & $(14,8)$ \\
\hline Tem tido a ideia de acabar com a vida & 1 & $(1,8)$ \\
\hline Se sente uma pessoa inútil, sem préstimo & 3 & $(5,5)$ \\
\hline
\end{tabular}

Fonte: Elaboração própria.

\section{Discussão}

Neste estudo, a proporção de DPM foi de 24,1\%. Em estudo realizado com trabalhadores da saúde da Bahia foi encontrada uma prevalência de $21,0 \%{ }^{(17)}$. Já em estudo realizado no Rio Grande do Sul, a prevalência encontrada foi de $33,7 \%{ }^{(12)}$. Este último revelou que existem duas vezes mais chances de os trabalhadores terem as capacidades reduzidas para o trabalho quando existe alta prevalência para suspeição dos $\mathrm{DPM}^{(12)}$.
$\mathrm{Na}$ amostra investigada, os DPM mostraram associação significativa com a variável sexo. Deve-se considerar que, além de a população ser predominantemente feminina, a suspeição de DPM foi evidenciada apenas nas mulheres. No perfil histórico da enfermagem, há o predomínio do sexo feminino, o que ainda ocorre nos dias atuais. A literatura descreve que grande parte das mulheres enfrenta a dupla jornada de trabalho, considerando a segunda no ambiente doméstico, com altas exigências, tanto quanto no 
ambiente profissional, o que aumenta a sobrecarga psicológica ${ }^{(18)}$.

Ao considerar-se as características dos trabalhadores em estudo, observou-se proporção mais alta de DPM nas trabalhadoras casadas ou com união estável e com filhos. A carga de trabalho adicional imposta à mulher trabalhadora em seu ambiente individual, associada ao ambiente de trabalho desgastante, favorece a ocorrência de danos à saúde mental em decorrência da demanda excessiva ${ }^{(18)}$.

Evidenciou-se, nesta investigação, que apenas auxiliares e técnicos de enfermagem apresentaram DPM. Este resultado é compatível com o exposto em outras pesquisas, que também encontraram maior prevalência de DPM nessa categoria comparada aos enfermeiros ${ }^{(12,19)}$. Alguns fatores podem explicar esse resultado, a exemplo de o enfermeiro possuir maior controle sobre seu processo de trabalho, devido às atividades gerenciais que executa, além de ocupar posição mais reconhecida no serviço e na sociedade ${ }^{(20)}$.

Observou-se ainda que os indivíduos da amostra em estudo apresentaram nível de instrução mais alto do que o requerido para as atividades que exercem, isto é, mesmo atuando como auxiliares ou técnicos de enfermagem, possuem graduação e especialização. Considera-se este fato um elemento que pode acarretar sentimentos de desvalorização e desmotivação, tendo em vista que possuem conhecimento além do que é exigido para as atividades que desempenham $^{(12)}$.

No que tange ao tempo de trabalho na instituição, foi identificada maior prevalência de DPM nos trabalhadores que ali atuavam há mais de 20 anos. Desse modo, o tempo prolongado de trabalho pode ser considerado um predisponente ao adoecimento do trabalhador. Estudos afirmam que a exposição constante ao ambiente de trabalho estressante, com alta demanda de trabalho e baixo reconhecimento, favorece, no longo prazo, o adoecimentodo do trabalhador, podendo manifestar-se como acomentimentos físicos e psicológicos ${ }^{(8,21)}$.

Em relação ao turno de trabalho, os resultados contrariam as evidências, que sugerem o trabalho noturno como gerador de adoecimento $^{(21)}$. Isso pode ser explicado pelas características desses setores no período noturno, que prestam assistência apenas às cirurgias de urgência, reduzindo. assim, a demanda de trabalho.

A baixa demanda psicológica no trabalho do $\mathrm{BC}$ pode ser considerada um aspecto benéfico para esses trabalhadores, posto que altas demandas aumentam o risco de desequilíbrio entre vida pessoal e profissional. Em contrapartida, o baixo controle foi evidenciado como significativo em relação aos DPM, sendo um elemento prejudicial para o desenvolvimento laboral, pelo fato de poder produzir perda de habilidade e desmotivação para realizar as tarefas ${ }^{(22)}$.

O baixo apoio social é um fator que tem sido reconhecido como prejudicial para os trabalhadores, visto que estudos recentes realizados na área de enfermagem constataram que é um fator importante para contrabalançar os efeitos negativos que o trabalho pode causar nos indivíduos $^{(23-24)}$.

No que se refere aos grupos de sintomas, observou-se maiores prevalências no grupo sintomas somáticos e humor depressivo/ansioso. A maior frequência desses fatores pode ser considerada como reflexo do mundo contemporâneo, principalmente em setores que envolvem alta densidade tecnológica, onde o excesso de informações simultâneas podem agravar as respostas somáticas ${ }^{(25)}$.

Nesse sentido, ainda é importante salientar o cenário no qual as categorias de enfermagem desenvolvem seu trabalho. Nesses ambientes as demandas psicológicas são significativas, em decorrência do convívio com sofrimento e condições precárias de trabalho. Esses fatores são predisponentes às alterações emocionais desses trabalhadores, provocando sintomas negativos e potencialmente causadores do adoecimento psicológico $^{(4)}$.

Considerou-se como limitações do estudo a possibilidade do viés do trabalhador sadio, pois a investigação foi realizada no local de trabalho. O número reduzido da amostra investigada também não permite a generalização dos resultados. Do mesmo modo, a coleta dos dados pelo 
autorrelato pode subestimar as reais particularidades dos respondentes.

\section{Conclusão}

Conclui-se que os resultados evidenciaram a proporção de 24,1\% para os DPM nos trabalhadores de enfermagem do referido $\mathrm{BC}$; quanto aos fatores associados, o sexo feminino e o controle no trabalho apresentaram associação significativa com o desfecho.

As variáveis que tiveram maior frequência de DPM foram sexo feminino, idade entre 40-49 anos, casados ou em união estável, ensino superior, auxiliares e técnicos de enfermagem, mais de 20 anos de trabalho na instituição e renda de 4-7 salários mínimos.

Apesar de os DPM não apresentarem associação significativa com grande parte das variáveis investigadas, esses estiveram presentes em aproximadamente um em cada quatro trabalhadores. Desse modo, devem-se levar em consideração os efeitos prejudiciais que o adoecimento mental pode causar para o indivíduo e a sociedade, tendo em vista que esses trabalhadores são prestadores de cuidados de saúde. Assim, é evidente a necessidade de implementação de medidas que promovam a saúde no trabalho, com melhorias organizacionais, investimentos em redes de apoio que busquem favorecer os relacionamentos interpessoais, além de viabilizar estratégias que promovam o desenvolvimento pessoal, no intuito de prevenir o adoecimento mental.

Espera-se que este estudo possa contribuir com o avanço do conhecimento científico no âmbito da enfermagem, e a promoção de melhorias nas condições de trabalho para os que atuam no ambiente hospitalar, em específico os trabalhadores de enfermagem do BC.

\section{Colaborações:}

1. concepção, projeto, análise e interpretação dos dados: Lauren Leal Ferreira, Evelin Daiane Gabriel Pinhatti e Renata Perfeito Ribeiro;
2. redação do artigo e revisão crítica relevante do conteúdo intelectual: Lauren Leal Ferreira, Evelin Daiane Gabriel Pinhatti, Cinthya Kallyane Gonçalves Queiroz e Renata Perfeito Ribeiro;

3. aprovação final da versão a ser publicada: Lauren Leal Ferreira, Evelin Daiane Gabriel Pinhatti, Cinthya Kallyane Gonçalves Queiroz e Renata Perfeito Ribeiro.

\section{Referências}

1. Merlo ARC, Bottega CG, Perez KV. Apresentação. In: Merlo ARC, Bottega CG, Perez KV, organizadores. Atenção à saúde mental do trabalhador: sofrimento e transtornos psíquicos relacionados ao trabalho. Porto Alegre: Evangraf, 2014. p. 8-11 [Internet]. [cited 2018 Aug 20]. Available from: http://cippto. com/wp-content/uploads/2017/03/atenca__ completo.pdf

2. Sousa VFS, Araújo TCCF. Estresse ocupacional e resiliência entre profissionais de saúde. Psicol Ciênc Prof [Internet]. 2015 [cited 2018 Sep 3];35(3):900-15. DOI: https://doi.org/10.1590/1982-370300452014. Available from: http://www.scielo.br/pdf/pcp/ v35n3/1982-3703-pcp-35-3-0900.pdf

3. Alves AP, Pedrosa LAK, Coimbra MAR, Miranzi MAS, Hass VJ. Prevalência de transtornos mentais comuns entre profissionais de saúde. Rev Enferm UERJ [Internet]. 2015 [cited 2018 Sep 3];23(1):64-9. DOI: https://doi.org/10.12957/reuerj.2015.8150. Available from: http://www.e-publicacoes. uerj.br/index.php/enfermagemuerj/article/ view/8150/12330

4. Teixeira CAB, Gherardi-Donato CAB, Pereira ECS, Cardoso SS. Estresse ocupacional e estratégias de enfrentamento entre profissionais de enfermagem. Enferm Glob [Internet]. 2016 Oct [cited 2018 Aug 20];44:299-309. Available from: http://scielo.isciii. es/pdf/eg/v15n44/pt_administracion3.pdf

5. Souza VS. Qualidade de vida dos profissionais de enfermagem atuantes em setores críticos. Rev Cuid [Internet]. 2018 [cited 2018 Sep 5];9(2):2177-86. DOI: http://dx.doi.org/10.15649/cuidarte.v9i2.506. Available from: https://www.revistacuidarte.org/ index.php/cuidarte/article/view/506

6. Lögde A, Rudolfsson G, Broberg RR, RaskAndersen A, Walinder R, Arakelian E. I am quitting my job. Specialist nurses in perioperative context and their experiences of the process and reasons to quit their job. Int J Qual Health Care [Internet]. 
2018 [cited 2018 Sep 5];30(4):313-20. DOI: https:// doi.org/10.1093/intqhe/mzy023

7. Silva ROC, Batista KM, Grazziano ES. Personalidade resistente nas equipes médica e de enfermagem em centro cirúrgico. Rev SOBECC [Internet]. 2014 [cited 2018 Sep 5];19(4):214-8. DOI: 10.5327/ Z1414-4425201400040007. Available from: https:// www.researchgate.net/publication/300082481_ Personalidade_resistente_nas_equipes_medica_e_ de_enfermagem_em_centro_cirurgico

8. Tostes MFP, Silva AQ, Garçon TL, Maran E, Teston EF. Dualidade entre satisfação e sofrimento no trabalho da equipe de enfermagem em centro cirúrgico. Rev SOBECC [Internet]. 2017 [cited 2018 Sep 25];22(1):3-9. DOI: https://doi.org/10.5327/ Z1414-4425201700010002. Available from: https:// revista.sobecc.org.br/sobecc/article/view/123/pdf

9. Battié RN, Rall H, Khorsand L, Hill J. Addressing perioperative staff member fatigue. AORN J [Internet]. 2017 [cited 2018 Sep 20];105(3):285-91. DOI: $10.1016 /$ j.aorn.2017.01.003

10. Machado RR, Gelbcke FL. Que brumas impedem a visibilização do centro de material e esterilização? Texto Contexto Enferm [Internet]. 2009 [cited 2018 Aug 25];18(2):347-54. DOI: http://dx.doi. org/10.1590/S0104-07072009000200019. Available from: http://www.scielo.br/pdf/tce/v18n2/19.pdf

11. Mari JJ, Williams P. A validity study of a psychiatric screening questionnaire (SRQ-20) in primary care in the city of Sao Paulo. Br J Psychiatry [Internet]. 1986 jan [cited 2018 Sep 15];48:23-6. DOI: https:// doi.org/10.1192/bjp.148.1.23

12. Magnago TSBS, Prochnow A, Urbanetto JDS, Bitencourt P, Beltrame M, Luz EMF. Relação entre capacidade para $O$ trabalho na enfermagem $e$ distúrbios psíquicos menores. Texto Contexto Enferm [Internet]. 2015 [cited 2018 Sep 15];24(2):362-70. DOI: http://dx.doi.org/10.1590/0104-07072015002580013. Available from: http://www.scielo.br/pdf/tce/v24n2/ pt_0104-0707-tce-24-02-00362.pdf

13. Rodrigues EP, Rodrigues US, Oliveira LMM, Laudano RCS, Nascimento Sobrinho CL. Prevalence of common mental disorders in nursing workers at a hospital of Bahia. Rev Bras Enferm [Internet]. 2014 [cited 2018 Sep 15];67(2):296-301. DOI: https://doi.org/10.5935/0034-7167.20140040. Available from: http://www.scielo.br/pdf/reben/ v67n2/0034-7167-reben-67-02-0296.pdf

14. Mattos AIS, Araújo TM, Almeida MMG. Interaction between demand-control and social support in the occurrence of common mental disorders. Rev Saúde Pública [Internet]. 2017 [cited 2018 Sep 15];51:48. DOI: https://doi.org/10.1590/ s1518-8787.2017051006446. Available from: http://www.scielo.br/pdf/rsp/v51/pt_0034-8910rsp-S1518-87872017051006446.pdf

15. Theorell T, Karasek RA. Current issues relating to psychosocial job strain and cardiovascular disease research. J Occup Health Psychol [Internet]. 1996 Jan [cited 2018 Sep 20];1(1):9-26. Available from: https://www.ncbi.nlm.nih.gov/ pubmed/?term=Current+issues + relating + to + psychosocial+job+strain+and+cardiovascular+ disease+research

16. Alves MGDM, Chor D, Faerstein E, Lopes CDS, Werneck GL. Short version of the "job stress scale": a Portuguese-language adaptation. Rev Saúde Pública [Internet]. 2004 [cited 2018 Sep 18];38(2):164-71. DOI: https://doi.org/10.1590/ S0034-89102004000200003. Available from: http:// www.scielo.br/pdf/rsp/v38n2/19774.pdf

17. Araújo TM, Mattos AIS, Almeida MMG, Santos KOB. Aspectos psicossociais do trabalho e transtornos mentais comuns entre trabalhadores da saúde: contribuições da análise de modelos combinados. Rev Bras Epidemiol [Internet]. 2016 [cited 2018 Sep 20];19(3):645-57. DOI: https://doi.org/10.1590/1980-5497201600030014. Available from: https://www.scielosp. org/article/ssm/content/raw/?resource_ssm_ path $=/$ media/assets/rbepid/v19n3/1980-5497rbepid-19-03-00645.pdf

18. Rotenberg L, Costa AS, Griep RH. Mental health and poor recovery in female nursing workers: a contribution to the study of gender inequities. Rev Panam Salud Publica [Internet]. 2014 [cited 2018 Sep 25];35(3):179-85. Available from: https:// scielosp.org/pdf/rpsp/2014.v35n3/179-185

19. Pai DD, Lautert L, Souza SBC, Marziale MHP, Tavares JP. Violence, burnout and minor psychiatric disorders in hospital work. Rev Esc Enferm USP. [Internet]. 2015 [cited 2018 Sep 25];49(3):457-64. DOI: https://doi.org/10.1590/ S0080-623420150000300014. Available from: http://www.scielo.br/pdf/reeusp/v49n3/00806234-reeusp-49-03-0460.pdf

20. Lage CEB, Alves MS. (Des)Valorização da enfermagem: implicações no cotidiano do enfermeiro. Enferm Foco [Internet]. 2016 [cited 2018 Sep 25];7(3/4):12-6. DOI: https:// doi.org/10.21675/2357-707X.2016.v7.n3/4.908. 
Available from: http://search.ebscohost.com/login . as px?direct $=$ true $\&$ site $=$ eds -1 ive $\& d b=$ a9h\&AN=121292827

21. Rosado IVM, Russo GHA, Maia EMC. Produzir saúde suscita adoecimento? As contradições do trabalho em hospitais públicos de urgência e emergência. Ciênc Saúde Colet [Online]. 2015 [cited 2018 Sep 25];20(10):3021-32. DOI: https:// doi.org/10.1590/1413-812320152010.13202014. Available from: http://www.scielo.br/pdf/csc/ v20n10/1413-8123-csc-20-10-3021.pdf

22. Ng LP, Chen IC, Ng HF, Lin BY, Kuar LS. Influence of job demands and job control on work-life balance among Taiwanese nurses. J Nurs Manag [Internet]. 2017 Sep [cited 2018 Sep 27];25(6):43848. DOI: https://doi.org/10.1111/jonm.12482. Available from: https://www.ncbi.nlm.nih.gov/ pubmed/28419626

23. Zurlo MC, Vallone F, Smith AP. Effects of individual differences and job characteristics on the psychological health of Italian nurses.
Eur J Psychol [Internet]. 2018 [cited 2018 Sep 27];14(1):159-75. DOI: https://doi.org/10.5964/ ejop.v14i1.1478. Available from: https://ejop. psychopen.eu/article/view/1478/pdf

24. Adriaenssens J, Hamelink A, Bogaert PV. Predictors of occupational stress and well-being in First-Line Nurse Managers: A cross-sectional survey study. Int J Nurs Stud [Internet]. 2017 May [cited 2018 Sep 27];73:85-92. DOI: https://doi.org/10.1016/j. ijnurstu.2017.05.007

25. Santos RR, Greco PBT, Prestes FC, Kirchhof RS, Magnago TSBS, Oliveira MA. Sintomas de distúrbios psíquicos menores em estudantes de enfermagem. Rev baiana enferm [Internet]. 2016 [cited 2018 Sep 25];30(3):1-14. DOI: https://doi. org/10.18471/rbe.v30i3.16060. Available from: https://portalseer.ufba.br/index.php/enfermagem/ article/view/16060/pdf_66

Recebido: 30 de setembro de 2018

Aprovado: 30 de julho de 2019

Publicado: 13 de novembro de 2019

A Revista Baiana de Enfermagem utiliza a Licença Creative Commons - Atribuição-NãoComercial 4.0 Internacional.

https://creativecommons.org/licenses/by-nc/4.0/

Este artigo é de acesso aberto distribuído sob os termos da Licença Creative Commons (CC BY-NC). Esta licença permite que outros remixem, adaptem e criem a partir do seu trabalho para fins não comerciais. Embora os novos trabalhos tenham de lhe atribuir o devido crédito e não possam ser usados para fins comerciais, os usuários não têm de licenciar esses trabalhos derivados sob os mesmos termos. 